\title{
Towards a Service-Oriented Architecture framework for educational serious games
}

\author{
Maira B. Carvalho*,, Francesco Bellotti ${ }^{\dagger}$, Jun Hu*, Jannicke Baalsrud Hauge ${ }^{\ddagger}$, \\ Riccardo Berta ${ }^{\dagger}$, Alessandro De Gloria ${ }^{\dagger}$ and Matthias Rauterberg* \\ *Industrial Design, Eindhoven University of Technology, Netherlands \\ ${ }^{\dagger}$ ELIOS Lab, DITEN, University of Genoa, Italy

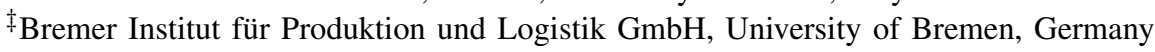

\begin{abstract}
Producing educational serious games can be costly and time-consuming. The Service-Oriented Architecture (SOA) approach of software development can offer a solution to reduce costs and foment serious games development. In this work, we apply a model called Activity Theory-based Model of Serious Games (ATMSG) for identifying existing relevant components that can be reused for different educational serious games. We apply the derived structure to classify the elements of an existing game and to identify how it can be refactored and expanded following the SOA paradigm.
\end{abstract}

Index Terms-Service-oriented architecture, serious games, educational serious games, serious games development, software engineering

\section{INTRODUCTION}

Educational serious games are designed to meet specific learning requirement. Consequently, such games are typically conceived as one-of-a-kind products fully customized according to the clients' requirements. Thus, main barriers for large scale deployment of serious games are the high production costs, challenging and time-consuming production process, and low reusability of the final product and its components [1]. Based upon well proven concepts of using Service-Oriented Architectures (SOA) for increasing the reusability by maintaining a high customization degree, it has been proposed that SOA can also benefit serious games development by reducing costs and time to market, while at the same time allowing customization in a relatively easy and reconfigurable way [2].

While there is a growing number of services for serious games [3], we are still lacking structures identifying relevant and usable serious games elements and how to interconnect these elements in the game. Consequently, if a group of serious game developers wants to take advantage of such services, there are questions on how to identify components suitable to be converted to services, taking into account that these services should have a high degree of reusability to serve several games.

The main objective of this paper is therefore to identify candidate serious games components that could be developed as services for games within different genres and domains. For that end, we apply a newly developed model which connects different components at different levels within a game, called Activity Theory-based Model of Serious Games (ATMSG) [4]. To show the suitability of our analysis to the development of educational serious games in practice, we apply our results to refactor and propose the expansion of an existing serious game to further exploit the SOA paradigm.

\section{A. Related work}

The adoption of SOA for serious games is currently limited [2], but there are examples of using SOA in entertainment games, particularly online multiplayer games, upon which we can draw, as the examples below show.

Houten and Jacobs [5] present an architecture for distributed multiplayer simulation games. However, even though originally an entertainment game, it can be used for training and learning purposes. Nevertheless, the architecture is restricted to the genre of simulation games; furthermore, the architecture does not explicitly support an environment in which several games share the same resources. Shaikh, Sahu, Rosu, et al. [6] describe the design of an on-demand service platform to enable sharing resources across online games. Their architectural solution focuses on solving problems of scaling the infrastructure in response to players' demand in massively multiplayer online games. BinSubaih and Maddock [7] use the SOA design philosophy to enable game portability across different game engines, with the objective of removing the strict dependency of a game to the engine underneath it. While not strictly a SOA, the work highlights well the benefits of separation of concepts and reusability in game design.

The previous mentioned works show the benefits of SOA for game development. However, they also show that there is still room for improvement, since what has been proposed so far is either restricted to a specific genre of games, or it solves one single aspect of architectural decisions in service-based game development.

To expand these works also for the development of educational serious games, we need to explicitly identify how learning elements can be incorporated in such architectures. This has to be done in a way that allows for reusability while still supporting the specific learning goals of the game. To reflect upon which serious games components can be reused in service format, we applied a model called Activity Theory-based Model of Serious Games (ATMSG), which we describe in the next section. 


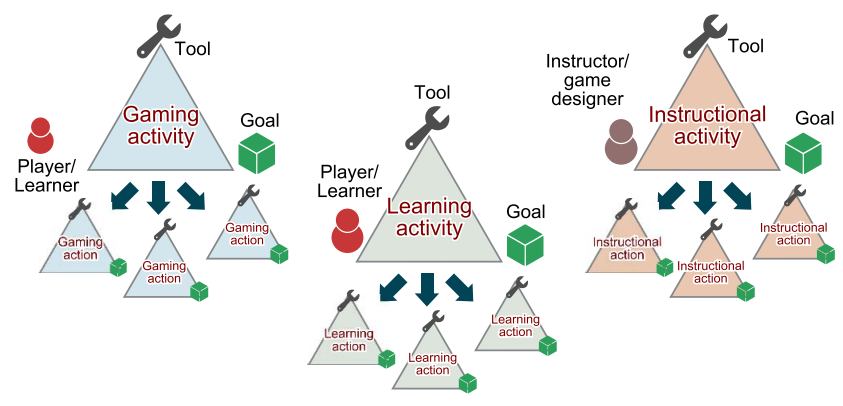

Fig. 1. Each activity is formed by a sequence of actions mediated by tools, with specific goals

\section{B. The Activity Theory-based Model of SGs}

The Activity Theory-based Model of Serious Games (ATMSG) [4] uses activity theory [8] to delineate a model that represents several different low-level components of an educational serious game as the game unfolds, and how these components are connected to the educational and entertainment high-level objectives of the game. In ATMSG, educational serious games are seen as used in the context of four activities: the gaming activity, the learning activity, the intrinsic instructional activity (performed inside the game) and the extrinsic instructional activity (performed by the instructor outside of the game).

In the model, these activities are further decomposed into a sequence of actions mediated by tools with specific goals, giving us a set of categories (Figure 1). These categories form the basis for a taxonomy of serious games components, in which existing taxonomies of learning, instruction, games and serious games are reorganized according to the ATMSG model [4]. Serious game components are understood as the pieces of an educational serious game - its actions, tools and goals - that constitute the gameplay over time, e.g. characters, tokens, tips, help messages, challenges, graphics, feedback mechanisms, assessment, etc.

The full taxonomy gave us an overlook of a large number of commonly found elements of serious games, which we could then analyze and classify in order to identify the possible components of a service-based serious game framework.

\section{IDENTIFYING REUSABLE SERIOUS GAMES ELEMENTS}

A crucial initial step in implementing Service-Oriented Architectures is decomposing the business domain into its functional areas, subsystems and desired goals, in order to identify processes and high-level business use cases. These processes are then selected as possible candidates for being implemented as services. This step is common among several methodologies for the development of SOA solutions (e.g. SOMA [9], SDLM [10] and SOAF [11]). In this section, we therefore provide such an initial analysis of the business domain.

From ATMSG's taxonomy of serious games elements, we collected a number of relevant items. The criteria for the selection were (a) relevance for the effectiveness of educational serious games, and (b) possibility of reuse across different games and learning domains, at least within the same game genre.

These items are discussed below, grouped by the activities according to the ATMSG model.

\section{A. Game activity}

Game engines (such as Unity) have been successfully used to abstract a number of gaming elements, particularly those related to building a game world and its rules. Nevertheless, even when game engines are used, games are typically still built in a way that does not reveal the game's inner workings. It is thus necessary to create a way to expose in-game events to external modules in a reusable manner, translating those events into information that will be useful to external modules.

Because the majority of gaming elements are highly related to game genre and topic, we did not specify gaming elements as candidates for being converted as services, but instead treated most of the gaming actions as events to which external modules can listen. The exception are actions related to obtaining information, which are game- and genre-independent, and thus excellent candidates for abstraction.

Among gaming tools, elements that are independent of the genre are those related to goal metrics and feedback on the goals, such as achievements, performance scores, leaderboards. And, in a related fashion, among gaming goals, the elements related to competition based on performance can be abstracted, particularly if existing social networks are used to connect players to their peers.

\section{B. Learning activity}

Learning activity elements are highly dependent on game genre and on the specific implementation of the serious game.

This is particularly true in the case of learning actions. For example, actions such as memorizing, locating, classifying or assessing will have particular implementations depending on both the topic and on the game itself. Consequently, no reusable elements were selected from this list for inclusion as candidate services.

Among learning tools, however, some elements were considered general enough to be useful for a subset of game genres, even if they are not be relevant to all kind of games at all times. In particular, surveys and questionnaires - similarly to knowledge bases - are tools that can be easily abstracted and implemented separately from the game. We also identified that stand-alone modules to store and display media (e.g. audio, video and pictures) can be developed. Finally, student diaries can also be implemented in a way that can be gameand topic-independent.

Lastly, among learning goals, the most genre- and topic- independent element is "learning how to learn", or self-reflection on the learning process. A module to provide students with an overview of their own progress is another good candidate for a reusable service for serious games. 


\section{Instructional activity}

Intrinsic instruction: The following intrinsic instructional actions were selected as candidate services: scaffolding, repetition, show similar problems and supporting recovery from errors. These elements are related to adapting the level of challenge to the player's current capabilities, which is an important factor for the efficacy of educational mediums [12].

Among intrinsic instructional tools, we highlighted quantitative assessment of performance, either using simple metrics (goal achievement, scores, etc.) or more complex methods such as the Competence-based Knowledge Space Theory [12].

From the list of intrinsic instructional goals, several items were selected: presenting the stimulus, providing feedback, assessing the performance, fostering confidence and providing satisfaction to the player. The items above relate to in-game assessment, feedback, and automatic adjustment of instruction (adaptivity).

Extrinsic instruction: Some elements in the extrinsic instruction list are similar to the ones already described in the intrinsic instructional activity. Here, however, the focus is on elements that a service-based framework could still incorporate to support the instructor in assessing and giving feedback to the student via the game.

Qualitative assessment of performance is an action that is both important and potentially relevant across serious games genres. It is the only item selected among the list of extrinsic instructional actions, since the other actions happen, by definition, outside of the game.

Among tools and goals, the selected items are also featured in the intrinsic instruction activity: performance measures, performance assessment and feedback. These elements were selected as candidates because they can be used to inform instructors about learners' performance, and then provide a way to incorporate the instructor's input back into the game.

In this section, we described our analysis of the educational serious game domain, in which we selected a list of elements that were considered both relevant and with a high potential for reuse. Table I summarizes the elements according to the activity to which they belong.

\section{REUSABLE COMPONENTS FOR A SERVICE-BASED ARCHITECTURE FOR SERIOUS GAMES}

Once the relevant educational serious games components described in the ATMSG taxonomy were identified and collected, we regrouped them according to their domains and functionalities, so that we could identify the clusters of reusable components to be implemented as services in a SOA framework for serious games. The result of this grouping is shown in the column "Functional domains" in Table I. For each of these functional domains, we identified a list of candidate functionalities that could be implemented as services, which are represented in Figure 2 and further described below.

- Between-players interaction: Services to collect, display and compare scores, such as social leaderboards.

- Student-instructor interaction: Services for querying players/students, prompting for answers to questions
TABLE I

ELEMENTS OF THE ATMSG TAXONOMY THAT COULD BE IMPLEMENTED AS SERVICES, WITH THEIR IDENTIFIED FUNCTIONAL DOMAINS

\begin{tabular}{|c|c|c|c|}
\hline Activity & $\begin{array}{l}\text { Element } \\
\text { type }\end{array}$ & Items & Functional domains \\
\hline \multirow{6}{*}{ Gaming } & \multirow[t]{3}{*}{ Actions } & Events listener & Game connectors \\
\hline & & $\begin{array}{l}\text { Watch / Listen to / } \\
\text { Read information }\end{array}$ & $\begin{array}{l}\text { Information storage and } \\
\text { retrieval }\end{array}$ \\
\hline & & Ask questions & $\begin{array}{l}\text { Information storage and } \\
\text { retrieval }\end{array}$ \\
\hline & \multirow[t]{2}{*}{ Tools } & Social network score & $\begin{array}{l}\text { Between-players } \\
\text { interaction }\end{array}$ \\
\hline & & Leaderboards & $\begin{array}{l}\text { Between-players } \\
\text { interaction }\end{array}$ \\
\hline & Goals & Competition & $\begin{array}{l}\text { Between-players } \\
\text { interaction }\end{array}$ \\
\hline \multirow{6}{*}{ Learning } & Actions & - & - \\
\hline & \multirow[t]{3}{*}{ Tools } & $\begin{array}{l}\text { Surveys, } \\
\text { questionnaires }\end{array}$ & $\begin{array}{l}\text { Student-instructor } \\
\text { interaction }\end{array}$ \\
\hline & & Student diary & $\begin{array}{l}\text { Student-instructor } \\
\text { interaction }\end{array}$ \\
\hline & & $\begin{array}{l}\text { Media assets (audios, } \\
\text { films, graphics, etc.) }\end{array}$ & $\begin{array}{l}\text { Information storage and } \\
\text { retrieval }\end{array}$ \\
\hline & \multirow[t]{2}{*}{ Goals } & Reflective observation & Feedback \\
\hline & & Learning how to learn & Feedback \\
\hline \multirow{9}{*}{$\begin{array}{l}\text { Intrinsic } \\
\text { instruction }\end{array}$} & \multirow[t]{4}{*}{ Actions } & $\begin{array}{l}\text { Quantitative } \\
\text { assessment }\end{array}$ & Assessment \\
\hline & & Scaffolding & Adaptivity \\
\hline & & $\begin{array}{l}\text { Show similar } \\
\text { problems }\end{array}$ & Adaptivity \\
\hline & & $\begin{array}{l}\text { Support recovery } \\
\text { from errors }\end{array}$ & Adaptivity \\
\hline & Tools & $\begin{array}{l}\text { Performance } \\
\text { measurements }\end{array}$ & Assessment \\
\hline & \multirow[t]{4}{*}{ Goals } & Assess performance & Assessment \\
\hline & & Provide feedback & Feedback \\
\hline & & Confidence & Assessment, Adaptivity \\
\hline & & Satisfaction & Assessment, Adaptivity \\
\hline \multirow{4}{*}{$\begin{array}{l}\text { Extrinsic } \\
\text { instruction }\end{array}$} & Actions & $\begin{array}{l}\text { Qualitative } \\
\text { assessment }\end{array}$ & Assessment \\
\hline & Tools & $\begin{array}{l}\text { Performance } \\
\text { measures }\end{array}$ & Assessment \\
\hline & \multirow[t]{2}{*}{ Goals } & Assess performance & Assessment \\
\hline & & Provide feedback & Feedback \\
\hline
\end{tabular}

(questions and surveys) or for reflections in their learning process (student diaries).

- Information storage and retrieval: For topics related to the knowledge inside the game (i.e. non-playing characters' knowledge about the game world) or about the learning domain itself, services to allow for management and use of this information can be useful. Descriptive metadata can facilitate resource discovery and subsequent reuse in different games. We also include in this category services that can connect to knowledge databases and convert this information to different formats that can be useful in the game (e.g. adapters for natural language 


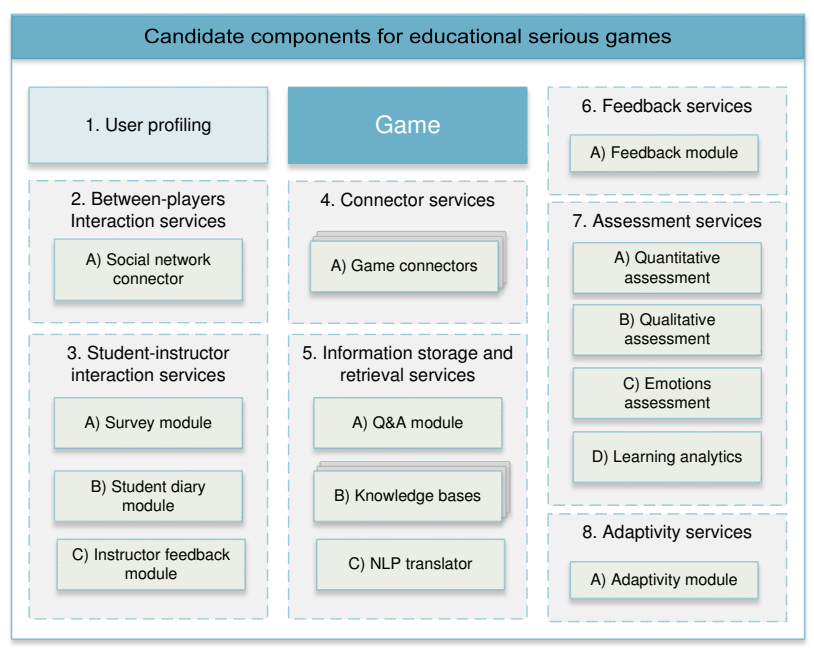

Fig. 2. The identified functional domains and their candidate components for implementation as services in a SOA framework for serious games

interaction or converters of knowledge bases to question and answer formats).

- Feedback: Feedback services provide a way to send the results of in-game assessment back to the game, in order to enable the provision of learning feedback, and to support the player's self-reflection on learning.

- Assessment: Assessment services can include modules for quantitative (automatic) and qualitative (instructor-provided) assessment, in addition to usage data that can help identify patterns of usage (learning analytics). It can also include modules for assessment of player/student's engagement, confidence and satisfaction.

- Adaptivity: Adaptivity services are responsible for consolidating information coming from several different assessments services, evaluating this information, making decisions on how the game should react, and serving this information back to the game.

- Game connectors: Game connector services provide adapter modules and data models that link external services to the game. This is possibly a game engine plug-in responsible for implementing trigger managers that detect important in-game events and forward messages to other services. These connectors will most likely be game- or at least genre-specific.

- User profiling: Although not directly derived from the taxonomy, a common user profile service is required in order to enable interaction, synchronization and persistent features across different games and learning settings.

The functional areas described above are an important asset to translate the theory, i.e. the ATMSG model, to the practical implementation of a SOA framework for serious games. Clustering elements allows us to identify relationships and to map information flows. This step will be invaluable to the development of a framework that enables reusability while still meeting the specific requirements of each serious game.

\section{AdOPTING SOA IN SERIOUS GAME DEVELOPMENT}

In the previous section, we identified the key functionalities of reusable components that are relevant for serious games development. In order to deliver a proof of concept, we applied our findings to propose the refactoring of an existing game, called "The Journey" [2]. The game is a serious game for teaching basic elements of probability to high school and entry-level university students, in which players have to understand how to calculate the probabilities of events and use their knowledge to make the best possible decisions along the way. "The Journey" implements some basic aspects of a Service-Oriented Architecture to provide adaptation features for learning. With this analysis, we show how the game can be expanded to further exploit the SOA paradigm.

In the current architecture of the game, two main functionalities are realized by an external service (called "CbKST service"): the assessment of the player's competences, and the computation and suggestion of the next appropriate task for the player's current abilities. These functionalities correspond to the items "quantitative assessment" and "adaptivity module". In addition, the service also realizes one auxiliary function, which is managing the current learning session. This can be considered a very simplified form of user profiling that exists simply as a way to connect a user to the results of his or her assessments in the game.

The functional domains and components described in the previous section help us in analyzing the game to identify possibilities for refactoring. Among the elements in Figure 2, items 7-A and 8-A (quantitative assessment and adaptivity) are already services in the current implementation. Elements 1, 5-A, 5-B and 6-A (user profiling, Q\&A module, knowledge base and feedback module), on the other hand, are current game functionalities, but not implemented as services. According to our analysis, these would be the best candidates for being converted to reusable services.

Another point to notice is that, in the current version of "The Journey", one external service (i.e. the "CbKST service") congregates two different functionalities, of two different (but related) domains: assessment and adaptivity. The problem with this combination is that the service, by treating both functionalities as one block, eliminates the possibility that other types of assessment (e.g. of the player's emotions) are incorporated in the decision model that provides the adaptation suggestions back to the game.

This case study shows us how migrating educational serious games to a SOA architecture based on the components proposed in this work can help developers in structuring their serious game in a way that promotes reusability and compositionality. For example, implementing the user profile as a service brings the immediate added benefit of decoupling the information about the player's achievements from the game itself, facilitating the use of this information by an instructor to provide feedback to the learner in an easier way. In addition, the use of knowledge bases for game tasks and learning content opens the possibility that other games and 
learning tools reuse the information. Finally, this structure helps developers in deciding the optimal level of granularity for the desired functionalities as to allow their easy integration within an overarching service architecture for serious games.

The downsides of applying this approach to serious game development involve the time and cost to refactor the code, and the increased complexity in how to test it. For this reason, it is crucial that the future service-oriented architecture framework for serious games includes not only the specifications of the services and interoperability standards, but also recommendations on how to minimize costs, and particularly how to perform quality assurance.

\section{CONCLUSION AND FUTURE WORK}

In this paper, we proposed a list of reusable components that are relevant for the development of educational serious games within different genres and domains, and which will provide the basis for a service-oriented architecture (SOA) for serious games. In order to generate this list of components and functionalities, we utilized a model called Activity Theory-based Model of Serious Games (ATMSG), which helped us address the specific requirements of educational serious games that current SOA frameworks for entertainment games do not cover. To illustrate how the components can be used in practice, we applied the list of components to analyze the current architecture of "The Journey", a SOA-based adaptive game, and to propose the directions for its future improvement.

By determining elements and functionalities being commonly used across educational serious games, we advanced one further step in the process of creating a SOA framework to support reusability of serious game components. This framework will simplify the serious game development process and thus lead to reduction of costs and time to market. It will also encourage the use of interoperability standards and a consistent structure across game components, increasing the overall quality of the process and of the final product.

The next step of this work is service specification: from the list of candidate services, we will categorize them into a service hierarchy, then describe in more detail the components that need to be implemented, such as required data, rules, configurable profiles, messaging, event management, interoperability standards, etc. The final objective of this effort is to create a service framework that will support the establishment of a whole ecosystem of services for educational serious games. In addition, this will serve as a roadmap for service development, indicating which are the critical components that need to be implemented in order to benefit a higher number of serious game developers.

\section{ACKNOWLEDGMENT}

This work was supported in part by the Erasmus Mundus Joint Doctorate in Interactive and Cognitive Environments, which is funded by the EACEA Agency of the European Commission under EMJD ICE FPA n 2010-0012. We thank the colleague Erik van der Spek for comments that greatly contributed to this work.

\section{REFERENCES}

[1] I. A. Stanescu, A. M. Stanescu, M. Moisescu, I. S. Sacala, A. Stefan, and J. Baalsrud Hauge, "Enabling interoperability between serious game and virtual engineering ecosystems," in International Design Engineering Technical Conferences and Computers and Information in Engineering Conference (ASME), Buffalo, New York, USA, 2014. DOI: 10.1115/DETC2014-35418.

[2] M. B. Carvalho, F. Bellotti, R. Berta, A. D. Gloria, G. Gazzarata, J. Hu, and M. Kickmeier-Rust, "A case study on service-oriented architecture for serious games," Entertainment Computing, vol. 6, pp. 1-10, 2015. DOI: 10.1016/j.entcom.2014.11.001.

[3] Serious Games Society, Serious games web services catalog, 2013. [Online]. Available: http :// services . seriousgamessociety.org/ (visited on 04/04/2014).

[4] M. B. Carvalho, F. Bellotti, R. Berta, A. De Gloria, C. Islas Sedano, J. Baalsrud Hauge, J. Hu, and M. Rauterberg, "An activity theory-based model for serious games analysis and conceptual design," Computers \& Education, 2015, (Online first). DOI: 10 . $1016 / \mathrm{j}$. compedu.2015.03.023.

[5] S.-P. A. van Houten and P. H. Jacobs, "An architecture for distributed simulation games," in Proceedings of the 36 th conference on Winter simulation, Winter Simulation Conference, 2004, pp. 2081-2086.

[6] A. Shaikh, S. Sahu, M.-C. Rosu, M. Shea, and D. Saha, "On demand platform for online games," IBM Systems Journal, vol. 45, no. 1, pp. 7-19, 2006. DOI: 10.1147/ sj.451.0007.

[7] A. BinSubaih and S. Maddock, "G-factor portability in game development using game engines," in Proceedings of the 3rd International Conference on Games Research and Development, 2007, pp. 163-170.

[8] Y. Engeström, Learning by Expanding: An Activity-theoretical Approach to Developmental Research. Orienta-Konsultit Oy, 1987.

[9] A. Arsanjani, S. Ghosh, A. Allam, T. Abdollah, S. Ganapathy, and K. Holley, "SOMA: a method for developing service-oriented solutions," IBM Systems Journal, vol. 47, no. 3, pp. 377-396, 2008. DOI: 10.1147/sj.473. 0377.

[10] M. P. Papazoglou and W.-J. Van Den Heuvel, "Service-oriented design and development methodology," International Journal of Web Engineering and Technology, vol. 2, no. 4, pp. 412-442, 2006.

[11] A. Erradi, S. Anand, and N. Kulkarni, "SOAF: an architectural framework for service definition and realization," in IEEE International Conference on Services Computing (SCC'06), 2006, pp. 151-158.

[12] M. D. Kickmeier-Rust and D. Albert, "Educationally adaptive: balancing serious games," International Journal of Computer Science in Sport, vol. 11, no. 1, pp. 1-10, 2012. 


\section{IEEE 15th International Conference on Advanced Learning Technologies}

ICALT 2015 


\section{Proceedings}

\section{IEEE 15th International Conference on Advanced Learning Technologies}

6-9 July 2015

Hualien, Taiwan

\section{Editors}

Demetrios G Sampson, University of Piraeus \& CERTH, Greece

Ronghuai Huang, Beijing Normal University, China

Gwo-Jen Hwang, National Taiwan University of Science \& Technology, Taiwan

Tzu-Chien Liu, National Taiwan Normal University, Taiwan

Nian-Shing Chen, National Sun Yat-sen University, Taiwan

Kinshuk, Athabasca University, Canada

Chin-Chung Tsai, National Taiwan University of Science \& Technology, Taiwan

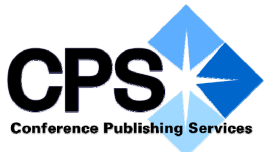

Los Alamitos, California

Washington • Tokyo

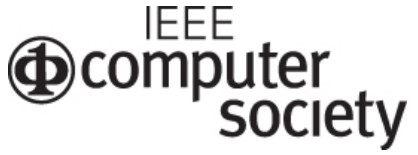


Copyright (C 2015 by The Institute of Electrical and Electronics Engineers, Inc.

\title{
All rights reserved.
}

Copyright and Reprint Permissions: Abstracting is permitted with credit to the source. Libraries may photocopy beyond the limits of US copyright law, for private use of patrons, those articles in this volume that carry a code at the bottom of the first page, provided that the per-copy fee indicated in the code is paid through the Copyright Clearance Center, 222 Rosewood Drive, Danvers, MA 01923.

Other copying, reprint, or republication requests should be addressed to: IEEE Copyrights Manager, IEEE Service Center, 445 Hoes Lane, P.O. Box 133, Piscataway, NJ 08855-1331.

The papers in this book comprise the proceedings of the meeting mentioned on the cover and title page. They reflect the authors' opinions and, in the interests of timely dissemination, are published as presented and without change. Their inclusion in this publication does not necessarily constitute endorsement by the editors, the IEEE Computer Society, or the Institute of Electrical and Electronics Engineers, Inc.

IEEE Computer Society Order Number E5547

BMS Part Number CFP15261-USB

ISBN 978-1-4673-7333-3

Additional copies may be ordered from:

\footnotetext{
IEEE Computer Society Customer Service Center 10662 Los Vaqueros Circle P.O. Box 3014 Los Alamitos, CA 90720-1314 Tel: + 18002726657

Fax: + 17148214641 http://computer.org/cspress csbooks@computer.org
}

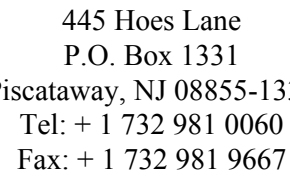
IEEE Service Center

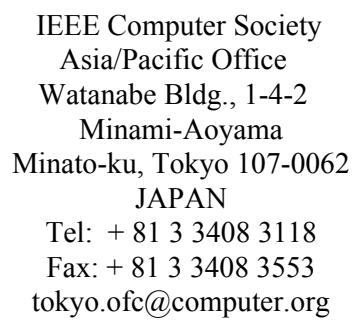

Individual paper REPRINTS may be ordered at: <reprints@computer.org>

Editorial production by Lisa O'Conner

Cover art production by Mark Bartosik

\section{(IEEE
Society}

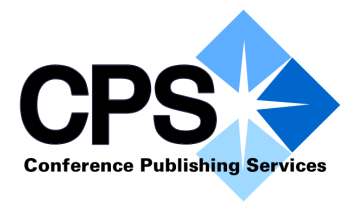

\author{
IEEE Computer Society \\ Conference Publishing Services (CPS)
}

http://www.computer.org/cps 


\section{IEEE 15th International Conference on Advanced Learning Technologies \\ ICALT 2015 \\ Table of Contents}

Preface

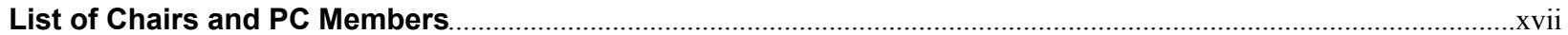

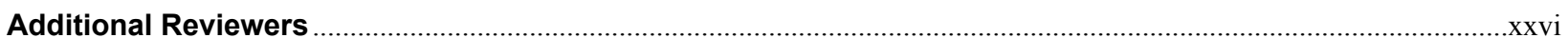

\section{Invited Papers}

Educational Games and the Design Based Research Approach .....

Miguel Nussbaum

Advancing the State of the Art in Advanced Learning Technologies: [Re-]Connecting Theory,

Research, Practice and Policy

J. Michael Spector

Technology and Knowledge Creation

Seng Chee Tan

\section{Track 1. Digital Systems for Open Access to Education and Learning}

SangeetKosh: An Open Web Platform for Music Education

Tejaswinee Kelkar, Anon Ray, and Venkatesh Choppella

Exploring Different Routes from LMS Towards PLE: A Dialectical Perspective

Vladimir Tomberg, Mart Laanpere, and Hans Põldoja

Designing and Developing Cloud-Based eBooks for Solving Teachers' Usage Barriers ...

I-Ching Chao, Po-Sheng Chiu, Chih-Chien Kao, Ying-Hung Pu, and Yueh-Ming Huang

Assessing Students' Learning Experience and Achievements in a Medium-Sized Massively

Open Online Course

Weichao Chen, Jiyou Jia, Jingmin Miao, Xiaomeng Wu, Aihua Wang, and Baijie Yang

Understanding Student Disaffection in Large-Scale Online Learning .......

Khe Foon Hew 


\section{Track 2. Adaptive and Personalised Technology-Enhanced Learning}

Classification-Based Approach to Concept Map Generation in Adaptive Learning

Xiaopeng Huang, Kyeong Yang, and Victor Lawrence

Towards an Integrative Learning Environment for Java Programming

Sebastian Gross and Niels Pinkwart

Online Tests Based on Contributions Provided by Teachers and Students during Face to Face

Lectures

Franck Silvestre, Philippe Vidal, and Julien Broisin

Constructing Collaborative Learning Groups with Maximum Diversity Requirements

Yulei Pang, Raymond Mugno, Xiaozhen Xue, and Huaying Wang

Indexing Learning Scenarios by the Most Adapted Contexts: An approach Based on

the Observation of Scenario Progress in Session

Mariem Chaabouni, Claudine Piau-Toffolon, Mona Laroussi, Christophe Choquet,

and Henda Ben Ghezala

Towards a Web-Based Adaptive Problem-Based Learning Application ....

Yongwu Miao, Disi Wang, Mbombui Nongho Fon-Pah, Mohammed Samaka, and Ulrich Hoppe

Modeling Learner's Emotions with PAD

Matthias Chan Yong Shun, Miao Chun Yan, An Bo, and Leung Cyril

Eye-Tracking Assisted Self-Directed Learning System

Eman Yasser Daraghmi, Chih-Tien Fan, Wei-Jun Lin, Zih-You Peng, and Shyan Ming Yuan

Students' Satisfaction in Learning Style-Based Adaptation .55 Mohammad Alshammari, Rachid Anane, and Robert J. Hendley

Using Knowledge Space theory to Personalize Teaching for Groups of Students .58

Rim Zakaria and Imran A. Zualkernan

Improving Communication and Project Management through an Adaptive Collaborative

Learning System .....

Jeffrey M. Kurcz, Ting-Wen Chang, and Sabine Graf

On Generic Communication Patterns between Courseware Objects in Extensible Learning

Support System Architecture for Self- and Group Learning Kiyoshi Nakabayashi and Yosuke Morimoto

Modelling Learner's Personality Profile through Analysis of Annotation Digital Traces

in Learning Environment

Nizar Omheni, Anis Kalboussi, Omar Mazhoud, and Ahmed Hadjkacem

Student Daily Practices Identification Algorithm for Mobile Learning Application

Neculai Ivascu, Maiga Chang, and Kristin Garn

PECALE: An Environment for Enhancing Personalization and Learner Engagement in

an Online Learning Platform

Betty Mayeku, Sviatoslav Edelev, Sunaina Prasad, Hemanth Karnal, and Dieter Hogrefe

A Boosting Algorithm for Auto-Generating Quiz for Traffic Education .

Jinn-Tser Lin, Wen-Ling Chen, Yu-Chieh Wu, and Jie-Chi Yang 
Towards an Educator-Centred Digital Teaching Platform: The Ground Conditions for

a Data-Driven Approach

Andrew Koster, Tiago Primo, Fernando Koch, Állysson Oliveira, and Hyunkwon Chung

An Analysis of Goal Orientation Pattern and Self-Efficacy for Explanation of Programming

Plans

Chung-Cheng Tseng, Po-Yao Chao, and K. Robert Lai

\section{Track 3. Wireless, Mobile, and Ubiquitous Technologies for Learning}

Career Support for International Students in Japan Using Ubiquitous Learning Log System

Noriko Uosaki, Hiroaki Ogata, Kousuke Mouri, and Erdenesaikahan Lkhagvasuren

Systematic Fusion of Gesture Sensors for Practical Learning in Virtual Environments

Matthias Weise, Raphael Zender, and Ulrike Lucke

A Web-Based Framework to Design and Deploy Mobile Learning Activities: Evaluating Its

usability, Learnability and Acceptance

Janosch Zbick, Isabella Nake, Marcelo Milrad, and Marc Jansen

Preliminary Research on Self-Regulated Learning and Learning Logs in a Ubiquitus Learning

Environment

Masanori Yamada, Chengjiu Yin, Atsushi Shimada, Kentaro Kojima, Fumiya Okubo,

and Hiroaki Ogata

Analysis of Ubiquitous-Learning Logs Using Spatio-Temporal Data Mining

Kousuke Mouri, Hiroaki Ogata, and Noriko Uosaki

Mobile Devices in English Language Instruction: Facilitation and Benefits

Gloria Shu Mei Chwo

A Dictionary of Gestures for Multitouch-Based Interactive Geometry Software

Helena M. Reis, Seiji Isotani, Isabela Gasparini, and Riichiro Mizoguchi

Technical Description of Equation Sensei: A Mobile-Assisted Learning Application

in Mathematics

Rex P. Bringula, John Nikko S. Alvarez, Maron Angelo C. Evangelista, Richard B. So,

and Marivic M. Gatus

ContactJS-A Cross-Platform Context Detection Framework

Tobias Moebert, Stefanie Lemcke, and Ulrike Lucke

A Low Cost, Portable Platform for Information Assurance and Security Education

Dan Chia-Tien Lo, Kai Qian, Wei Chen, and Tamara Rogers

The Development of a Decision Support System for Successful Mobile Learning

Po-Sheng Chiu, Chih-Chien Kao, Ying-Hung Pu, Pei-Fang Lo, and Yueh-Ming Huang

Informal Learning Behavior Analysis Using Action Logs and Slide Features in E-Textbooks

Atsushi Shimada, Fumiya Okubo, Chengjiu Yin, Kentaro Kojima, Masanori Yamada, and Hiroaki Ogata

The Effect of Signals in Hypertext Reading by Tablet Computers

Xiaoxia Zheng, Wei Cheng, Zhengcheng Fan, and Guang Chen 
Baby Boomers' Behaviors and Attitudes of Newspaper Reading: A Comparison among Three Layouts

Lin-Chao Fu and Hsiu-Ping Yueh

\section{Track 4. Digital Game and Intelligent Toy Enhanced Learning}

Math Detective: Digital Game-Based Mathematical Error Dectection, Correction and Explanation

Hercy N.H. Cheng, Yi-Ju Lin, Meng Wang, and Tak-Wai Chan

Participatory Design in EU-TOPIA: A Serious Game for Intercultural Competences

during Work Mobility

Hassina El Kechaï and Laëtitia Pierrot

Augmented Reality Laboratory for High School Electrochemistry Course

Ming-Puu Chen and Ban-Chieh Liao

Make and Play: Card Games as Tangible and Playable Knowledge Representation Boundary

Objects

Andrea Valente and Emanuela Marchetti

Musikinésia-An Educational Adventure Game for Keyboard Learning

Rogério Augusto Bordini, Joice Lee Otsuka, Delano Medeiros Beder, Lucas Ferreira Fonseca,

Pablo Augusto Gonçalves de Freitas, Antônio Avanzi Nunes, Daniel Lopes Santiago,

Glauber Lúcio Alves Santiago, and Marcia Rozenfeld Gomes de Oliveira

Towards a Service-Oriented Architecture Framework for Educational Serious Games

Maira B. Carvalho, Francesco Bellotti, Jun Hu, Jannicke Baalsrud Hauge, Riccardo Berta,

Alessandro De Gloria, and Matthias Rauterberg

Effectiveness of MMORPGS in Enhancing Student Interaction

Wong Seng Yue and H. Yeong Tze

An Investigation of a Game-Based Anti-Drug System: Addictive Learners vs. Non-addictive

Learners

Chang-Mai Huang, Jen-Hang Wang, and Sherry Y. Chen

Effects of Gesture-Based Fitness Games on Functional Fitness of the Elders .

Feng-Ru Sheu, Yun-Lin Lee, Hsiu-Tao Hsu, and Nian-Shing Chen

A Mobile Educational Game for Teaching Computer Architecture

Ahmed Tlili, Fathi Essalmi, and Mohamed Jemni

Urban Data Games: Creating Smart Citizens for Smart Cities

Annika Wolff, Gerd Kortuem, and Jose Cavero

Using NAO Humanoid Robot in Kindergarten: A Proposed System

Atheer Alkhalifah, Bashayer Alsalman, Deema Alnuhait, Ohoud Meldah, Sara Aloud,

Hend S. Al-Khalifa, and Hind M. Al-Otaibi

A Mobile-Phone Camera Text-Recognition Game as an Alternative Assessment in Vocabulary

Instruction for Learning Indonesian as a Foreign Language Classroom 168

Melissa Mustika, Chi-Te Kao, Antonius Siswanto, Shein-Yung Cheng, Jia-Sheng Heh,

and Cheng-Yuan Chang 


\section{Track 5. Computer Supported Collaborative Learning}

Towards Designing a Mobile Social Learning Application with Meaningful Gamification Strategies

Sei-Young Kim, Hyo-Jeong So, Soonmo Kwon, Seungjae Oh, Kyudong Park, Minjin Ko,

Jaewon Yoo, and Gyuhwan Oh

The Impact of Cross-Age Peer Tutors on Knowledge Elaboration, Knowledge Convergence, and Group Performance in Computer Supported Collaborative Learning

Lanqin Zheng

The Empirical Study on Self-Regulation, Co-Regulation, and Socially Shared Regulation

in Computer-Supported Collaborative Learning

Lanqin Zheng and Junhui Yu

Making Explicit the Moodle Instructional Design Language

Nour El Mawas, Lahcen Oubahssi, and Pierre Laforcade

Here's Looking at you, Kid - Can Gaze Awareness Help to Learn to Learn Together

in Collaborative Problem Solving?

Andreas Harrer, Christian Schlösser, Philipp Schlieker-Steens, and Andrea Kienle

A Qualitative Analysis on Mutual Engagement in CSCL Scenarios Using Eye-Tracking

Technology

Andreas Lingnau and Andreas Harrer

A Study on Collaborative and Competitive Strategies of Learners Used in an Educational Game

Vanessa Sissi Herbst

Online Peer Learning: What Influences the Students' Learning Experience

Songlak Sakulwichitsintu, Douglas Colbeck, Leonie Ellis, and Paul Turner

A Design Proposal for Learner-Centered Visualisations of Learning Analytics in Collaborative

Scenarios

Andreas Harrer

Formalization of Recurrent Uses of e-Learning Tools as Reusable Pedagogical Activities

Esteban Loiseau, Nour El Mawas, and Pierre Laforcade

Online Collaborative Learning for Improving Argumentation of Student with Different Levels

of Science Prior Knowledge

Yu-Ren Lin, Hsiao-Ching She, Wen-Tsung Yang, and Kai-Yi Huang

Helping Strategy Support in Synchronous Peer Tutoring System for Children

Mengping Tsuei

\section{Track 6. Technology-Enhanced Assessment in Formal and Informal Education}

Integrating Assessment into Augmented Reality-Based Learning Environments

María-Blanca Ibáñez, Diego Villarán, and Carlos Delgado-Kloos

Exploring Constructive Learning Activity in Online Programming Discussion Forums

I. Han Hsiao

Effects of Group and Task Size in Computer Supported Collaborative Marking

Manuel Matas and Jorge Villalon 
What Happened to Non-linear Narrative? A Pedagogical Reflection

Emanuela Marchetti and Andrea Valente

A Preliminary Study of Integrating Flipped Classroom Strategy for Classical Chinese Learning

Yi Hsuan Wang

Combining Mobile Devices with NFC Technology in a Test Assessment System

Tsung-Sheng Cheng, Yu-Chun Lu, Chun-Chieh Chang, and Chu-Sing Yang

Students' Perceptions about Assessment Using an e-Learning Platform Rosalina Bessa Babo, Ana Isabel Azevedo, and Jarkko Suhonen

Correlation of Grade Prediction Performance with Characteristics of Lesson Subject Shaymaa E. Sorour, Jingyi Luo, Kazumasa Goda, and Tsunenori Mine

Quality Evaluation of Web-Based Educational Software: A Systematic Mapping 250 Aparecida M. Zem Lopes, Laís Z. Pedro, Seiji Isotani, and Ig I. Bittencourt

A Formative Assessment Ontology for Students' Lab Reports in Lab Book 253 Hakim Mokeddem, Cyrille Desmoulins, and Chalal Rachid

Understanding Undergraduates' Information Literacy from their Facebook Usage 256 Kan Min Lin

Application of Combination Weighting Method to Weight Calculation in Performance

Evaluation of ICT 258 Chun Lu, Lili Li, and Di Wu

\section{Track 7. Big Data in Education and Learning Analytics}

Towards Textual Reporting in Learning Analytics Dashboards

A. Ramos-Soto, M. Lama, B. Vázquez-Barreiros, A. Bugarín, and M. Mucientes. S. Barro

Can You Tell If they're Learning? Using a Pedagogical Framework to Measure Pedagogical

Activity 265

Tim O'Riordan, David E. Millard, and John Schulz

Semantic Description of the Experience API Specification .268 Juan C. Vidal, Thomas Rabelo, and Manuel Lama

\section{Track 8. Technology-Enhanced Science, Technology, Engineering, and Math Education}

Equality: A Tool for Free-form Equation Editing

Stephen Cummins, Ian Davies, Andrew Rice, and Alastair R. Beresford

A Conductive Chemistry Online Argumentation Courseware with Website Exploring Support Kai-Yi Huang, Yi-Lin Cheng, Hsiao-Ching She, and Yu-Ren Lin

Stickipedia: A Search Engine and Repository for Explanatory Analogies

Varun Kumar, Savita Bhat, and Niranjan Pedanekar

Applying the Formal Concept Analysis to Introduce Guidance in an Inquiry-Based Learning

Environment

Michael A. Bedek, Simone Kopeinik, Bernd Prünster, and Dietrich Albert 
Assessing the Impact of Virtual Labs: A Case Study with the Lab on Advanced VLSI

Garima Ahuja, Anubha Gupta, Harsh Wardhan, and Venkatesh Choppella

A Case Study on LEGO Activity in Physics Class: Taking the "Rotational Kinetic Energy"

for Example

Qianxia Jing, Lingyu Yang, Menglu Jiang, Ruiying Huai, and Feng-Kuang Chiang

The Development of Simulation-Based Learning System for Binary Tree of Data Structures Ah-Fur Lai and Pei-Jeng Wu

Social Media as a Teaching and Learning Tool for In-class Q\&A Activities to Promote Learning

and Transform College Engineering Classroom Dynamics: The Case of Facebook

Fu-Yun Yu and Yu-Hsin Liu

A Feedback Effectiveness Oriented Math Word Problem E-Tutor for E-Learning Environment

Kyle Morton and Yanzhen Qu

Explore College Students' Cognitive Processing during Scientific Literacy Online Assessments

with the Use of Eye Tracking Technology

Pei-Yi Tsai, Ting-Ting Yang, and Hsiao-Ching She

Explore the Eye Movement Regarding the Cognitive Process of Online Optic Reasoning

Learning.

Chou Wen-Chi and She Hsiao-Ching

A Generic Model for the Group Formation Problem Using Constraint Logic Programming 307

Grace S. Tacadao and Ramon Prudencio S. Toledo S.J.

\section{Track 9. Technology Enhanced Language Learning}

A Preliminary Study on Taiwanese EFL Adolescents' Perceptions of Mobile-Assisted

Post-Reading Tasks

Ya-Wen Ho and Chih-Cheng Lin

Intelligent Tutoring System for Voice Conversion in English

Deepika P. Vinchurkar and M. Sasikumar

Construction of a Voice-Based Asynchronous Communication System Utilizing Speech

Recognition and Its Potential for EFL Learners' Speaking Ability: A Pilot Study

Yuichi Ono, Takumi Ishii, and Akio Ohnishi

Using Smartphone to Facilitate English Communication and Willingness to Communicate in

a Communicative Language Teaching Classroom

Bo-Ru Luo, Yu-Lun Lin, Nian-Shing Chen, and Wei-Chieh Fang

A Domain-Specific Modeling Specification of Visual Instructional Design Languages: A

Moodle Experimentation

Aymen Abedmouleh

L3MS: A Lightweight Language Learning Management System Using Mobile Web

Technologies .326

Reem A. Alamer, Hind M. Al-Otaibi, and Hend S. Al-Khalifa

Middle School English Teachers' Attitude and Motivation Towards ICT-Enabled Teaching

in China

Kwan C.M. Ming and Sophia H.X. Liu 


\section{Track 10. Motivational and Emotional Aspects in Technology-Enhanced Learning}

Towards a Japanese Language Learning Process Based on Japanese Dubbing-A Case Study

on University Students

Chun-Chia Wang

Affective States in CSCL Environments: A Systematic Mapping of the Literature

Rachel Carlos Duque Reis, Carla Lopes Rodriguez, Kamila Takayama Lyra,

Patrícia Augustin Jaques, Ig Ibert Bittencourt, and Seiji Isotani

Enhancing Student Engagement through Personalized Motivations

Athanasios Staikopoulos, Ian O'Keeffe, Bilal Yousuf, Owen Conlan, Eddie Walsh, and Vincent Wade

Learners' Level of Metacognitive Awareness and Its Relationship with Listening Performance

Yajun Zeng and Christine Goh

An Affective Learning Environment for Java

Ramón Zatarain Cabada, María Lucía Barrón Estrada, Francisco González Hernández, and Raúl Oramas Bustillos

Learning Personality Modeling for Regulating Learning Feedback

Matthias Chan Yong Shun, Miao Chun Yan, Shen Zhiqi, and An Bo

An Evaluation of the Learning Attitude and Motivation in a Language Learning Support System

Jingyun Wang and Takahiko Mendori

Validation of the Teacher Readiness for Online Learning Measure Min-Ling Hung

Developing Successful Novice International Faculty through a Mentoring Program .364 Gloria Robles and Ana Lidia Franzoni

The Effect of Using a Talking Head in Academic Videos: An EEG Study 367 Diana Díaz, Rafael Ramírez, and Davinia Hernández-Leo

\section{Track 11. Technology Enabled Learning of Thinking Skills}

Impact of Blender Based 3D Mental Rotation Ability Training on Engineering Drawing Skills Kapil Kadam and Sridhar Iyer

Using an Automatic Approach to Classify Reflective Language Learning Skills of ESL Students 375 Gary Cheng and Juliana Chau

Development of Engineering Design Competencies Using TELE-EDesC: Do the Competencies

Transfer?

Madhuri Mavinkurve and Sahana Murthy

Academic Writing Support System Using Bayesian Networks

Masaki Uto and Maomi Ueno

Using Eye-Tracking to Investigate the Different 3D Representation on Students' Mental Model

Construction

Sheng-Chang Chen, Hsiao-Ching She, and Mi-Shan Hsiao 


\section{Track 12. Recommender Systems for Learning}

Enhancing Learning Object Recommendations for Teachers Using Adaptive Neighbor Selection

Stylianos Sergis and Demetrios G. Sampson

A Utility-Based Semantic Recommender for Technology-Enhanced Learning

Andrea Zielinski

Would Linked MOOC Courseware Enhance Information Search?

Shang-Wen Li and Victor Zue

Towards the Effective Use of Available Educational Resources: Designing Adaptive

Hypermedia Environments for the Engineering Sciences

Simon Carolan, Guillaume Moreau, Morgan Magnin, and Francisco Chinesta

Knowledge Recommender: An Application Based on the Social Knowledge Network

for Knowledge Recommendation

Shan Li, Juan Zheng, and Qianxia Jing

Social Analytics Framework to Boost Recommendation in Online Learning Communities

Yanyan Li, Haogang Bao, Yafeng Zheng, and Zhinan Huang

Towards a Multi-label Classification of Open Educational Resources

Marcos Mouriño García, Roberto Pérez Rodríguez, Luis Anido Rifón, and Manuel Vilares Ferro

An Interactive Annotation System to Support the Learner with Web Services Assistance

Anis Kalboussi, Nizar Omheni, Omar Mazhoud, and Ahmed Hadj Kacem

Learnersourced Recommendations for Remediation

Shang-Wen "Daniel” Li and Piotr Mitros

\section{Track 13. Technology Supported Education for People with Disabilities}

Alternative Concepts for Accessible Virtual Classrooms for Blind Users

Wiebke Köhlmann and Ulrike Lucke

A Learning Game for Deaf Learners

Mohamed Ali Khenissi, Yosra Bouzid, Fathi Essalmi, and Mohamed Jemni

Effectiveness of an Immersive Virtual Environment (CAVE) for Teaching Pedestrian Crossing

to Children with PDD-NOS

Aimilia Tzanavari, Nefi Charalambous-Darden, Kyriakos Herakleous, and Charalambos Poullis

Effects of Somatosensory Video Games on Simple Reactions of Institutional-Dwelling Older

Adults with Mild-Cognitive Impairments

Mao Liou, Shang-Ti Chen, Hsiu-Chi Fu, and I-Tsun Chiang

A Series of Leap Motion-Based Matching Games for Enhancing the Fine Motor Skills

of Children with Autism

Gaoxia Zhu, Su Cai, Yuying Ma, and Enrui Liu

Making Linear Equations Accessible for Visually Impaired Students Using 3D Printing

Noha Al-Rajhi, Amal Al-Abdulkarim, Hend S. Al-Khalifa, and Hind M. Al-Otaibi

A Teacher Professional Development Program for Designing Inclusive Learning Experiences .....

Silvia Margarita Baldiris Navarro, Panagiotis Zervas, Ramón Fabregat, and Demetrios G. Sampson 


\section{Track 14. Smart Learning Environments}

Integrating SERVQUAL and Importance-Performance Analysis for Assessing Smart Campus

Service Quality: A Case Study of an English Training Programme in Vietnam

I-Chang Tsai and Chung-Han Yeh

Investigating the Effectiveness of Speech-to-Text Recognition Application on Learning

Performance in Traditional Learning Environment

Rustam Shadiev, Yueh-Min Huang, Wu-Yuin Hwang, and Narzikul Shadiev

Performance Analysis of Parallel Particle Swarm Optimization Based Clustering of Students 446

Kannan Govindarajan, David Boulanger, Jérémie Seanosky, Jason Bell, Colin Pinnell,

Vivekanandan Suresh Kumar, Kinshuk, and Thamarai Selvi Somasundaram

A Framework of Teaching and Learning with e-Textbooks in Smart Learning Environment

Lei Fan, Ting-Wen Chang, Ronghuai Huang, and Wei Cheng

e-Schoolbag in China-Exploring Research Evidence for Large Scale Deployment

of e-Textbooks and Services

Tore Hoel

Design of Attention-Based Recommendation Learning Mechanism in the Cloud Computing

Environment . .456

Hong-Ren Chen and Ju-Hong Chen

Heuristic-Based Automatic Online Proctoring System .458

R.S. Vishnu Raj, S. Athi Narayanan, and Kamal Bijlani

Supporting Cognitive Skills of People Suffering from Dementia through a Sensor-Based System

Anastasios Karakostas, Ioulietta Lazarou, Georgios Meditskos, Thanos G. Stavropoulos,

Ioannis Kompatsiaris, and Magda Tsolaki

Towards Bringing Adaptive Micro Learning into MOOC Courses

Geng Sun, Tingru Cui, Kuan-Ching Li, Dongming Xu, Shiping Chen, Jun Shen, and William Guo

\section{Track 15. Virtual Worlds in Education and Training}

Teaching Interpersonal Problem Solving Skills Using Roleplay in a 3D Virtual World

for Special Education: A Case Study in Second Life 464

Anders I. Mørch, Melissa D. Hartley, and Valentina Caruso

Mind and Body Learn Together: Embodied Cognition and Language Learning

Yu-Ju Lan, Nian-Shing Chen, Yao-Ting Sung, and Tzu-Chien Liu

Teaching Renewable Energy Sources Using 3D Virtual World Technology

Foteini Grivokostopoulou, Isidoros Perikos, Kovas Konstantinos, and Ioannis Hatzilygeroudis

\section{Track 16. Knowledge Management in e-Learning}

Validating Algorithmic Optimization of Patient Allocation at Medical Schools: Which Patient

is the Best Fit for Undergraduate Training?

Felix Balzer, Martin Dittmar, Olaf Ahlers, and Niels Pinkwart

Exploring the Key Factors for Cooperate Implementation of Mobile Technology

Ming-Hsin Lu, Hsiu-Ping Yueh, and Weijane Lin 
Trends in E-Learning Research from 2002-2013: A Co-citation Analysis

Xiao-Fan Lin and Qintai Hu

A Competency-Based Model to Bridge the Gap between Academic Trainings and Industrial

Trades

Rémi Venant, Césdric Teyssié, Daniel Marquié, Philippe Vidal, and Julien Broisin

\section{Track 17. Large Scale Implementation of Technology Supported Educational} Innovations

Flipping, Engaging, and Teaming, Oh My! Lessons Learned from a Large Scale Curriculum

Reform at a US Medical School

Weichao Chen, Mary Kate Worden, and Elizabeth Bradley

\section{Track 18. Doctoral Consortium on Advanced Learning Technologies}

The Effects of Progress Bars on Diverse Learning Styles in Web-Based Learning

Te-Lien Chou and Sufen Chen 